\title{
(6) OPEN ACCESS \\ Long-term outcome after topical ciclosporin in severe dry eye disease with a 10-year follow-up
}

\author{
Morgane Straub, ${ }^{1}$ Alain M Bron, ${ }^{2,3}$ Aurore Muselier-Mathieu, ${ }^{2}$ \\ Catherine Creuzot-Garcher ${ }^{2,3}$
}

\begin{abstract}
${ }^{1}$ Department of Ophthalmology, University Hospital, Lyon Sud, France ${ }^{2}$ Department of Ophthalmology, University Hospital, Dijon, France ${ }^{3}$ Eye and Nutrition Research Group, CSGA, UMR 1324 INRA, 6265 CNRS, Burgundy, Dijon, France
\end{abstract}

\section{Correspondence to} Professor Catherine CreuzotGarcher, Department of Ophthalmology, University Hospital, CHU le Bocage, 14 rue Gaffarel Dijon 21000, France; Catherine.creuzotgarcher@chu-Dijon.fr

Received 26 April 2015 Revised 16 November 2015 Accepted 10 January 2016 Published Online First 28 January 2016

\section{CrossMark}

To cite: Straub M Bron AM, MuselierMathieu A, et al. Br J Ophthalmol

2016;100:1547-1550.

\section{ABSTRACT}

Aim To report a 10-year follow-up of patients suffering from severe dry eye syndrome (DES) initially treated with topical ciclosporin A (tCSA) for 6 months.

Methods The charts of 26 patients with severe DES related to keratoconjunctivitis sicca (KCS) and followed for a minimum 10-year follow-up were retrospectively reviewed. All of them were treated initially with tCSA for 6 months. The Schirmer I test, fluorescein and lissamine green staining scores and tear film break-up time (TBUT) were recorded to assess clinical symptoms before, during and after treatment. The subjective signs were evaluated with the ocular surface disease index (OSDI) questionnaire. Prolongation and reintroduction of tCSA after the initial treatment and combined treatments were also noted.

Results Overall the median (IQR) duration of tCSA treatment was 23 (7-51) months after a prolonged induction treatment lasting $20(8-41)$ months during the 10-year follow-up. For symptoms, a statistically significant difference in the OSDI between baseline and the end of the 10-year follow-up was not found $(p=0.67)$. We noted a statistically significant improvement in all clinical signs after the initial treatment period, still present at the end of follow-up. Only $6.5 \%$ of the patients needed reintroduction of tCSA after their prolonged induction treatment.

Conclusions The improvement observed after an initial tCSA treatment was sustained after a long-term followup with few cases requiring additional tCSA treatment. A prolonged induction treatment to decrease initial inflammatory local signs is a promising option in KCS.

\section{INTRODUCTION}

Dry eye disease (DED) is a frequent disease with a prevalence reaching $15 \%$ after 65 years of age. Women are particularly impacted after menopause because of hormonal status modifications. ${ }^{1}$ DED is associated with symptoms of ocular discomfort such as burning, foreign body sensation, dryness, grittiness, ocular pain, photophobia, blurred vision and visual fatigue. ${ }^{2}$ Keratoconjunctivitis sicca (KCS) is the most severe presentation of DED with subjective symptoms and objective signs related to ocular surface impairment. Several factors have been identified in DED: inflammation plays a major role with an increased expression of inflammatory markers, namely HLA-DR, intracellular adhesion molecules, inflammatory cytokines (interleukin (IL) 1, IL-6, IL-8), tumour necrosis factor and increased activity of metalloproteinases in the tear fluid. ${ }^{3}$ First-line treatments for KCS are palliative and do not treat the underlying cause of the disease. Unlike artificial tears, topical ciclosporin A (tCSA) works to restore the ocular surface, allowing increased production of tears by inhibiting the T-cell activation pathway. As an immunomodulatory agent, ciclosporin A, acting through the cellmediated inflammatory cascade, can reduce dry eye syndrome (DES). ${ }^{4}$ In two multicentre, randomised, prospective, phase III trials, tCSA $0.05 \%$ was shown to increase Schirmer values, reduce corneal staining, improve subjective symptoms and reduce the dependence on artificial tears. ${ }^{5}$ Therefore, ciclosporin has been shown to be an effective therapeutic agent for moderate-to-severe dry eye and was approved for this indication in December 2002 in the USA. The purpose of this retrospective study was to report the outcome of patients treated with an initial 6-month induction phase with tCSA 0.05\% (Restasis, Allergan, Buckinghamshire, UK) followed by an 'as-needed' tCSA treatment period during a 10 -year follow-up.

\section{PATIENTS AND METHODS \\ Patients}

Patients suffering from moderate-to-severe KCS were enrolled in a clinical trial conducted by Allergan (Study 192371-501) in May 2002 to assess the efficacy and the safety of tCSA in patients with moderate-to-severe dry eye. The study followed the Declaration of Helsinki guidelines for research involving human subjects and was approved by the local institutional review board. Once included, all the patients were treated for at least 6 months according to the design of the trial (period A). At the end of this trial, patients presenting persistent clinical signs and symptoms of dryness, tCSA was maintained through a temporary use authorisation according to the examiner's decision (period B). In some cases, tCSA was temporarily stopped after period B and then reintroduced (period C). Gender, age and duration of ocular dryness at inclusion as well as associated systemic diseases were collected. For this retrospective study, only patients with a minimum follow-up lasting 10 years were studied (see figure 1). All the patients were followed during this 10 -year period in the Department of Ophthalmology, Dijon University Hospital, Dijon, France. The inclusion criteria were the Schirmer I test $\leq 5 \mathrm{~mm} / 5 \mathrm{~min}$ in both eyes at baseline, tear break-up time (TBUT) $\leq 10 \mathrm{~s}$ (average of three tests) and fluorescein score $>2$ (Oxford Scheme) in both eyes. To be included, patients had to have felt uncomfortable for at least 3 months with a minimum of two symptoms among the following: feeling of dryness, foreign body sensation, burning or stinging. Exclusion criteria were the presence of uncontrolled systemic disease, active 


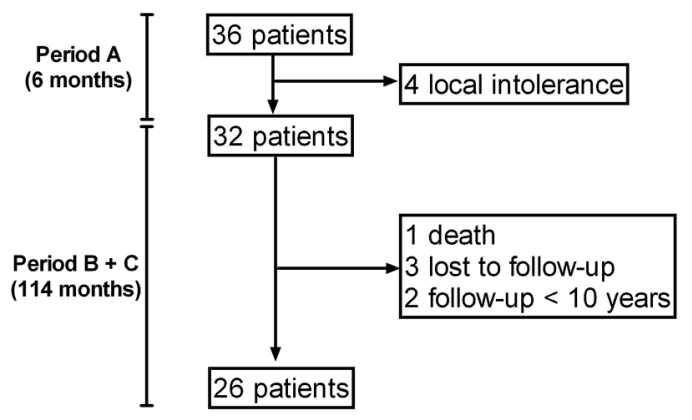

Figure 1 Flow chart of the 'Long-term outcome after topical cyclosporine in severe dry eye disease with a 10-year follow-up' study.

ocular infection or non-KCS ocular inflammation, known allergy or sensitivity to the study medications or its components, pregnancy, previous photorefractive surgery, abnormal lid position and contact lens wear.

\section{Clinical examination}

A complete ophthalmic examination with visual acuity, ocular pressure measurement and biomicroscopy was performed by a single examiner (CC-G). Patients presenting meibomitis (mild, moderate or severe) were identified. Corneal and interpalpebral conjunctival staining, TBUT and Schirmer test results were successively collected. The subjective symptoms were evaluated using the ocular surface disease index (OSDI) score and the number of artificial tear eye-drops or ointment was recorded. For corneal fluorescein staining, the entire cornea was examined using the slit lamp with blue cobalt illumination and a yellow barrier filter. Staining was graded using the Oxford Scheme 6-point scale, from 0 to 5 , using the same set of photographs as a guide. ${ }^{5}$ Lissamine green was then instilled, and interpalpebral conjunctival staining was evaluated, between $30 \mathrm{~s}$ and 2 min after instillation. Staining was graded using white light of moderate intensity, on interpalpebral regions of temporal and nasal conjunctiva, using the same Oxford Scheme. The sum of corneal and interpalpebral staining was therefore on a 0 -point to15-point scale. A negative change from baseline indicated an improvement. TBUT was performed with a slit lamp at $\times 10$ magnification using blue cobalt illumination. The Schirmer test I (without anaesthesia) was graded in millimetres after 5 min. A positive change from baseline indicated improvement. These clinical tests were performed before, during and after tCSA treatment and at each visit during the follow-up. Subjective

\section{Table 1 Patient characteristics at baseline}

\begin{tabular}{lc}
\hline Sex (female) & $25(96.2)$ \\
Age (years) & $60(57-66)$ \\
Sjögren disease associated with dry eye & $22(84.6)$ \\
Duration of ocular dryness (months) & $48(29-56)$ \\
Schirmer I test (mm) & $1(0-4)$ \\
Fluorescein staining score (Oxford scale) & $3(3-4)$ \\
Lissamine green staining score (Oxford scale) & $7(6-8)$ \\
Break-up time (s) & $3(3-4)$ \\
Ocular surface disease index (OSDI) score & $60.4(50.0-73.4)$ \\
Global number of drops per day & $6(5-10)$ \\
\hline Categorical variables are displayed as number (percentage). & \\
Continuous variables are displayed as median (IQR).
\end{tabular}

symptoms were graded with the OSDI questionnaire. ${ }^{6}$ A negative change from baseline indicated improvement.

\section{Treatment}

During the 6-month initial period, patients were asked to use tCSA $0.5 \%$ twice a day. They were allowed to use concomitant artificial tear eye-drops if needed. At the end of this initial period (period A), if relieved, patients could stop tCSA and use only artificial tears as needed. In other cases, tCSA was prolonged and prescribed twice daily as long as necessary (period B) to obtain a significant improvement of signs and symptoms according to the examiner's decision. In some cases, after a temporary stop, tCSA was reintroduced during the follow-up (period C) if needed. This was done through a temporary compassionate use of this drug since this drug was not commercially available in France at that time.

We recorded the total duration of tCSA treatment (period A $+B+C$ ), the number of patients requiring a prolongation of tCSA after period $\mathrm{A}$ and the duration of the prolonged induction period $\mathrm{A}+\mathrm{B}$, the number of patients who needed a reintroduction of tCSA after period $A+B$ and the number of patients till under tCSA at the end of the follow-up. We also recorded the number of additional treatments per day (before and during the follow-up), especially steroids, artificial teardrops and ointments as well as the side effects related to all the treatments.

\section{STATISTICAL ANALYSIS}

We considered only one eye per patient for analysis. We used non-parametric tests because the variables were not normally distributed (D'Agostino and Pearson omnibus normality test). Descriptive statistics are given as the median (IQR) for continuous variables and number (percentage) for categorical variables. For comparisons, we used the paired Wilcoxon test for continuous variables and the Fisher exact test or $\chi^{2}$ test for dichotomous data. The Spearman test was used for non-parametric correlations. The Prism V.5.01 software (Graphpad Software, La Jolla, California, USA) was used and $\mathrm{p}$ values $<0.05$ were considered statistically significant. All the tests were two tailed.

\section{RESULTS}

A total of 36 patients were included during period A, and 26 patients were ultimately followed for 10 years (figure 1). The characteristics of the patients are displayed in the table 1.

Twenty-two patients had Sjögren syndrome. One patient suffered from sarcoidosis and another one from primitive biliary cirrhosis. Two patients presented xerostomia and xerophthalmia with KCS but did not meet all the criteria for Sjögren disease according to Vitali et al. ${ }^{7}$ Sixty-three per cent of the patients presented associated meibomitis defined as mild (23.1\%), moderate $(30.2 \%)$ or severe $(15.4 \%)$. At baseline, the median OSDI score was $60.4(50.0-73.4)$ and $48.9(33.3-64.6)$ after the 10-year follow-up $(p=0.15)$. The Schirmer I test improved from 1.0 $(0.0-4.0) \mathrm{mm}$ at baseline to $2.5(1.2-3.0) \mathrm{mm}$ at 6 months without any statistical difference $(p=0.12)$. At the end of the 10-year follow-up, the median Schirmer I test reached 3.0 (2.0$5.0) \mathrm{mm}$ and was significantly improved compared with baseline $(p=0.02)$. The fluorescein staining score was significantly lowered, from $3.0(3.0-4.0)$ at baseline to $2.5(1.2-3.0)$ after 6 months $(p<0.001)$ and $2.0(1.1-3.0)$ after the 10 -year follow-up $(\mathrm{p}<0.001)$. The lissamine green conjunctival staining score decreased significantly from $7.0(6.0-8.0)$ at baseline to 6.0 $(3.5-7.0)$ at 6 months $(p=0.01)$ and after 10 years of follow-up $5.0(4.0-6.0)(\mathrm{p}<0.001)$. The TBUT increased from $3.0(3.0-$ 
$4.0)$ at baseline to $4.0(3.0-5.0)$ seconds at 6 months $(p=0.99)$ and rose to $4.0(3.0-6.0) \mathrm{s}$ at the 10 -year follow-up $(\mathrm{p}=0.03)$.

After a 10-year follow-up, the median duration of tCSA treatment was 23 (7-51) months (period $A+B+C$ ). All the patients still needed prolonged treatment with tCSA after the first 6 months. The duration of prolonged induction treatment $(A+B)$ without any tCSA discontinuation was 20 (8-41) months. After this initial tCSA treatment period $(\mathrm{A}+\mathrm{B})$, only $6.5 \%$ needed reintroduction of tCSA (period C), after a median delay of 40 (25-54) months. Only two patients were still taking tCSA after the 10 -year follow-up. The median time without any tCSA treatment was 24 (11-53) months during the whole 10-year period.

The overall number of topical lubricant eye-drops per day was significantly lower during the tCSA treatment compared with baseline, $5(3-6)$ vs $6(5-10)$ eye-drops $(p=0.001)$. The number of corticosteroid eye-drops per day decreased significantly from baseline to 6 months and 10 years, $1.5(1-3), 0.5(0.0-2.0)$ and 0 $(0-0)$ eye-drops $(p=0.001$ and $p=0.04$, respectively). Less ointment application was used at the end of the initial 6-month tCSA treatment $0(0-0)$ vs $1(0-1)(\mathrm{p}=0.01)$, but a statistical difference was no longer found after 10 years $0.5(0-1)(p=0.19)$.

Local adverse effects were noted throughout the follow-up for $15 \%$ of patients: $10 \%$ presented with a burning sensation at instillation, $3 \%$ a stinging sensation at instillation and $2 \%$ red eyes immediately after instillation. Only two patients temporarily interrupted tCSA treatment because of local side effects, during a short period of 1.5 (1-2) months, but tCSA treatment was rather well tolerated after reintroduction in these patients.

\section{DISCUSSION}

DES is a common disorder that is on the rise and is responsible for a significant impact on quality of life even in people with good vision, and can be considered as a public health problem. ${ }^{8}$ The diagnosis is based upon symptoms of ocular discomfort and/or objective clinical signs. In 2007, the International Dry Eye Workshop (DEWS) categorised dry eye into four levels of severity and established treatment guidelines based on disease severity. ${ }^{10} 11$ The management of KCS encompasses two main approaches. The non-pharmacological approach includes eyelid hygiene, avoidance of exacerbating factors and punctual plugs in selected cases. The pharmacological approach includes artificial tears, oral antibiotics (tetracyclines) in selected cases, topical corticosteroids and tCSA. Anti-inflammatory therapies are recommended beginning at level 2 (level 2 is characterised by both moderate symptoms and objective conjunctival and corneal staining). ${ }^{12}$ Several randomised studies have shown the efficacy of tCSA in DED. ${ }^{5} 13$ Indeed, inflammation plays a key role in dry eye pathogenesis. The inflammatory cell infiltration of the ocular surface leads to an increased expression of adhesion molecules and inflammatory cytokines. ${ }^{14}$ CSA was discovered in the early 1970 s as an antifungal agent; it is a neutral, hydrophobic, cyclic metabolite of the fungi Tolypocladium inflatum and Beauveria nevus. ${ }^{15}$ Through its immunomodulatory effect, tCSA specifically blocks T-cell activation and prevents synthesis of several proinflammatory cytokines. ${ }^{5} 1617$ Therefore, it decreases ocular inflammation and restores ocular surface integrity, with increased tear production. ${ }^{18}$ In contrast to topical corticosteroids, which carry the main risks of secondary glaucoma and cataract, ${ }^{19}$ the good safety profile of tCSA is appropriate for long-term therapy. ${ }^{20}$

In the present study, clinical tests showed an objective improvement after tCSA treatment. We found a significant improvement of fluorescein and the lissamine green staining score during the tCSA treatment compared with baseline during the first
6 months of use. When we compared baseline results and those obtained after the 10-year follow-up, with a median period of 23 months with tCSA treatment followed by a median period of 24 (11-53) months with no tCSA, we found a significant improvement for the Schirmer test, fluorescein and lissamine green staining scores and TBUT. These results are in accordance with Sall et al, ${ }^{5}$ who, in their large multicentre randomised phase III clinical trial, found that tCSA was effective in the treatment of moderate-to-severe chronic dry eye. Wilson and Perry ${ }^{21}$ also suggested the long-term effect of tCSA after a 1-year follow-up in DES. In our study, only $6.5 \%$ of our patients had tCSA reintroduced after the prolonged induction phase. Indeed, almost all of our patients needed prolonged tCSA treatment with a median duration of 23 months. Other studies have demonstrated that tCSA might require several months to produce a therapeutic effect and up to 6 months for maximum improvement. ${ }^{19}$

In this series, the OSDI score decreased by $10.5 \%$ at the 10 -year follow-up. In a large study with 5884 patients, Stonecipher et al ${ }^{22}$ found similar outcomes with a statistically significant improvement of dry eye symptoms after tCSA treatment, with reductions in the impact of KCS in daily activities. The overall number of eye-drops used by our patients was significantly decreased during the tCSA treatment period and at the end of the 10-year follow-up. This was similar for topical corticosteroids.

In our study, most of the patients presented associated meibomitis and showed a good clinical improvement with tCSA. In their study, Rubin et $a l^{23}$ reported that tCSA should have a positive impact on meibomitis, with an improvement of the viscosity of meibomian gland secretion, TBUT and Schirmer scores compared with a control group (tobramycin $0.3 \%$ /dexamethasone $0.1 \%$ ).

Overall in our series, tCSA was well tolerated with few patients presenting side effects. However, the primary Sjögren syndrome present in most patients can be associated with trigeminal sensory neuropathy, responsible for a decreased corneal and conjunctival sensation, which can explain this low side effect rate. ${ }^{24}$ Some patients reported non-specific side effects such as burning, stinging and red eyes at instillation. Interestingly, this occurred only in a later period. This could be related to recovery of corneal sensation after tCSA treatment. ${ }^{24}$ Indeed, Liang et $a l^{25}$ studied the safety of tCSA and concluded that tCSA was generally well tolerated, with no significant side effects. Considering the entire population treated initially, among the 36 patients, 4 needed to stop the treatment for a local side effect at an early stage and 2 patients secondarily experienced poor tolerance at instillation but did not stop tCSA for safety reasons.

These results suggest that tCSA with at least a 6-month initial treatment and afterwards a 23-month median treatment duration could have a sustained effect on dry eye symptoms and could slow down disease progression, without significant side effects. Although reported in the literature, ${ }^{26}$ this hypothesis needs to be confirmed with more robust findings in further series. While most patients required a limited period of tCSA treatment, a few patients were still under treatment at the end of this long follow-up, although none of them needed continuous treatment for 10 years (range 7-51 months). To predict which patient will need prolonged treatment remains challenging, but early treatment with ciclosporin in moderate-to-severe cases resistant to well-conducted treatments before fibrosis occurs seems a good option.

The patients included in this study can be considered as having relatively severe DES. The baseline clinical presentation associated with well-identified underlying systemic disease $(85 \%$ of patients presented Sjögren-associated DES) can be considered as 
worsening conditions. However, the fact that almost all our patients had Sjögren syndrome can also probably explain the good efficacy of ciclosporin because the tissue damage is due to cell-mediated injury. ${ }^{27}{ }^{28}$ The relevance of such an improvement of DED remains to be assessed from a clinical point of view. Indeed, DED is characterised by a poor correlation between signs and symptoms. ${ }^{29}$ In our series, the improvement of the fluorescein score remains moderate, varying from 3 at baseline to 2 after 10 years, for a mean $20 \%$ improvement that can be considered as clinically relevant. The statistically non-significant improvement of symptoms through OSDI results can be partly explained by the frequent corneal hypoesthesia found in severe DES. Therefore, a good indirect reflection of efficacy was the decreased number of lubricant eye-drops and steroid use by the patients.

We acknowledge several limitations to this study. First, the small size of the sample and the retrospective design of the study undertaken in one centre are real weaknesses. Second, a potential confounding factor is the common use of systemic treatments such as steroids and immunosuppressive drugs that can interfere with symptoms. While these treatments were requested to be stable for at least 6 months at inclusion, it is likely that these treatments varied at least for certain periods during such a long follow-up. However, the impact of systemic treatments is difficult to evaluate on local inflammatory signs. Third, the absence of a control group is another caveat and the question can be raised as to whether or not this improvement observed after such a long follow-up corresponded to spontaneous progression. However, all these patients belonged to the most severe group defined by the Delphi score with a pre-existing optimal treatment and regular use of steroid eye-drops. ${ }^{12}$ The fact that these patients with severe ocular surface diseases should have improved spontaneously with a subsequently decreased number of lubricant eye-drops is quite unlikely, but due to the limitations of the design of our study, we cannot draw definitive conclusions. Moreover, from an ethical point of view, it would have been impossible to leave these patients with such severe disease without treatment or with treatment such as steroids that have potential side effects for such a long time. Fourth, we have only considered patients with severe dry eye, thus limiting the number of include patients and the conclusions, which could have been drawn from other subgroups.

The strength of this study is the long follow-up. To our knowledge, this is the first time that such a long follow-up has been reported with tCSA. Our aim was to assess the efficacy of a tCSA treatment in durably improving ocular surface conditions. Another goal was to determine which information should be delivered to a patient suffering from severe DED and aiming to begin treatment with tCSA. This could be summarised as follows: the initial treatment with tCSA should be longer than 6 months. Local side effects related with tCSA use should be explained in details to the patients in order to improve a long-term compliance.

In conclusion, tCSA treatment decreased the objective clinical signs of KCS and was associated in a long-term perspective with an improvement of symptoms on everyday activities and a reduction of artificial tear use.

Contributors Design and conduct of the study (AMB, CC-G); collection management, analysis and interpretation of the data (MS, AMB, AM-M, CC-G). preparation, review or approval of the manuscript (MS, AMB, AM-M, CC-G).

Competing interests AMB: consultant for Allergan, Bausch Lomb, Théa, research grant Horus; CC-G: consultant for Allergan, Bayer, Bausch Lomb, Novartis, Théa, research grant Horus.

Patient consent Obtained.

Ethics approval Local institutional review board (CPP Est).
Provenance and peer review Not commissioned; externally peer reviewed.

Open Access This is an Open Access article distributed in accordance with the Creative Commons Attribution Non Commercial (CC BY-NC 4.0) license, which permits others to distribute, remix, adapt, build upon this work non-commercially, and license their derivative works on different terms, provided the original work is properly cited and the use is non-commercial. See: http://creativecommons.org/ licenses/by-nc/4.0/

\section{REFERENCES}

1 Lemp MA. Report of the national eye institute/industry workshop on clinical trials in dry eyes. CLAO J 1995;21:221-32.

2 Pflugfelder SC. Prevalence, burden, and pharmacoeconomics of dry eye disease. Am J Manag Care 2008;14:S102-6.

3 Smith VA, Rishmawi $\mathrm{H}$, Hussein $\mathrm{H}$, et al. Tear film MMP accumulation and corneal disease. Br J Ophthalmol 2001;85:147-53.

4 Yüksel B, Bozdağ B, Acar M, et al. Evaluation of the effect of topical cyclosporine A with impression cytology in dry eye patients. Eur J Ophthalmol 2010;20:675-9.

5 Sall K, Stevenson OD, Mundorf TK, et al. Two multicenter, randomized studies of the efficacy and safety of cyclosporine ophthalmic emulsion in moderate to severe dry eye disease. CsA Phase 3 Study Group. Ophthalmology 2000;107:631-9.

6 Schiffman RM, Christianson MD, Jacobsen G, et al. Reliability and validity of the Ocular Surface Disease Index. Arch Ophthalmol 2000;118:615-21.

7 Vitali C, Bombardieri S, Jonsson R, et al. Classification criteria for Sjogren's syndrome: a revised version of the European criteria proposed by the American-European Consensus Group. Ann Rheum Dis 2002;61:554-8.

8 Moss SE, Klein R, Klein BE. Incidence of dry eye in an older population. Arch Ophthalmol 2004;122:369-73.

9 Trattler W, Katsev D, Kerney D. Self-reported compliance with topical cyclosporine emulsion $0.05 \%$ and onset of the effects of increased tear production as assessed through patient surveys. Clin Ther 2006;28:1848-56.

10 No authors listed]. Design and conduct of clinical trials: report of the Clinical Trials Subcommittee of the International Dry Eye WorkShop (2007). Ocul Surf 2007:5:153-62.

11 Novack GD. Pharmacologic treatments for dry eye: a worthwhile investment? Cornea 2002;21:4-5.

12 Behrens A, Doyle JJ, Stern L, et al. Dysfunctional tear syndrome: a Delphi approach to treatment recommendations. Cornea 2006:25:900-7.

13 Rao SN. Topical cyclosporine $0.05 \%$ for the prevention of dry eye disease progression. J Ocul Pharmacol Ther 2010;26:157-64.

14 Perry HD, Donnenfeld ED. Dry eye diagnosis and management in 2004. Curr Opin Ophthalmol 2004;15:299-304.

15 Utine CA, Stern M, Akpek EK. Clinical review: topical ophthalmic use of cyclosporin A. Ocul Immunol Inflamm 2010;18:352-61.

16 Kunert KS, Tisdale AS, Stern ME, et al. Analysis of topical cyclosporine treatment of patients with dry eye syndrome: effect on conjunctival lymphocytes. Arch Ophthalmol 2000;118:1489-96.

17 Turner K, Pflugfelder SC, Ji Z, et al. Interleukin-6 levels in the conjunctival epithelium of patients with dry eye disease treated with cyclosporine ophthalmic emulsion. Cornea 2000;19:492-6.

18 Tatlipinar S, Akpek EK. Topical ciclosporin in the treatment of ocular surface disorders. Br J Ophthalmol 2005;89:1363-7.

19 Pflugfelder SC. Antiinflammatory therapy for dry eye. Am J Ophthalmol 2004;137:337-42.

20 Lemp MA. Management of dry eye disease. Am J Manag Care 2008;14:S88-101.

21 Wilson SE, Perry HD. Long-term resolution of chronic dry eye symptoms and signs after topical cyclosporine treatment. Ophthalmology 2007;114:76-9.

22 Stonecipher K, Perry HD, Gross RH, et al. The impact of topical cyclosporine A emulsion $0.05 \%$ on the outcomes of patients with keratoconjunctivitis sicca. Curr Med Res Opin 2005:21:1057-63.

23 Rubin M, Rao SN. Efficacy of topical cyclosporin $0.05 \%$ in the treatment of posterior blepharitis. J Ocul Pharmacol Ther 2006;22:47-53.

24 Toker $E$, Asfuroğlu E. Corneal and conjunctival sensitivity in patients with dry eye: the effect of topical cyclosporine therapy. Cornea 2010;29:133-40.

25 Liang $\mathrm{H}$, Baudouin C, Daull $\mathrm{P}$, et al. Ocular safety of cationic emulsion of cyclosporine in an in vitro corneal wound-healing model and an acute in vivo rabbit model. Mol Vis 2012;18:2195-204.

26 Rao SN. Reversibility of dry eye deceleration after topical cyclosporine $0.05 \%$ withdrawal. J Ocul Pharmacol Ther 2011:27:603-9.

27 Sacchetti M, Mantelli F, Lambiase A, et al. Systematic review of randomised clinical trials on topical ciclosporin A for the treatment of dry eye disease. $\mathrm{Br} J$ Ophthalmol 2014;98:1016-22.

28 Zhang $X$, Schaumburg CS, Coursey TG, et al. CD8(+) cells regulate the T helper-17 response in an experimental murine model of Sjögren syndrome. Mucosal Immunol 2014:7:417-27.

29 Nichols KK, Nichols JJ, Mitchell GL. The lack of association between signs and symptoms in patients with dry eye disease. Cornea 2004;23:762-70. 